\title{
Results from the Herschel Gould Belt Survey in the Ophiuchus Main Cloud
}

\author{
Bilal Ladjelate ${ }^{1}$, Philippe André ${ }^{1}$, Vera Könyves ${ }^{1}$, \\ Alexander Men'shchikov ${ }^{1}$, and the HGBS team ${ }^{2}$ \\ ${ }^{1}$ Service d'Astrophysique, AIM, CEA Saclay, 91191, Gif-sur-Yvette, France \\ email: bilal.ladjelate@cea.fr, ${ }^{2}$ http://gouldbelt-herschel.cea.fr
}

\begin{abstract}
As part of the Herschel Gould Belt Survey (HGBS), the Ophiuchus molecular cloud was imaged in the submillimeter range. Here, we summarize and briefly discuss the main results.
\end{abstract}

Keywords. stars: formation, mass function, ISM: structure

\section{Introduction}

Our understanding of star formation has greatly advanced in the past 10 years thanks to the help of large photometric multi-wavelength surveys. In particular, the Herschel Gould Belt Survey (André et al. 2010) represents a significant step forward towards a better understanding of the processes happening in nearby molecular clouds.

As part of the HGBS, extensive submillimeter continuum images of the Ophiuchus Main Cloud (L1688) were produced. Harbouring low-mass star formation, at $140 \mathrm{pc}$, the Ophiuchus starforming region has been studied for more than three decades at wavelengths ranging from the X-ray (Montmerle et al.1983) to the infrared and millimeter domains (e.g. Wilking et al.1989, Motte et al.1998). A deep census of both prestellar cores and young protostars was obtained from the Herschel data using the multi-scale, multi-wavelength source extraction algorithm, getsources (Men'shchikov et al.2012). One of the advantages of Herschel is that it observed at wavelengths covering the peak of the spectral energy distributions (SEDs) of young protostars and prestellar cores.

\section{Main Results}

In the L1688 cloud, we found approximately 200 candidate prestellar cores, including 70 cores with a robust classification, and $\sim 200$ young stellar objects including 40 embedded protostars. Among the starless cores detected with Herschel, the densest objects are gravitationally bound and can be considered prestellar in nature. In addition to prestellar cores, Herschel also detects very low-mass unbound cores (Fig. 1).

Nearly all $(99 \%)$ detected prestellar cores are found in zones at $A_{V} \geqslant 8$ (Fig. 2), and $80 \%$ of them are detected in filaments with column densities above $5 \times 10^{21} \mathrm{H}_{2} . \mathrm{cm}^{-2}$ as estimated by getfilaments.

Filaments are ubiquitous structures in star-forming regions (André et al.2014, Könyves et al.2015) and their close correlation with the presence of prestellar cores testifies to their importance in the early stages of star-formation. The detection of filaments in L1688 (Fig. 3) with different algorithms, such as DisPerSe (Sousbie 2011) and getfilaments (Men'shchikov 2013), is an important result in a region not previously known to be filamentary (cf. Wilking et al.1989).

After deriving the properties of the extracted Herschel cores, we obtained the distribution of prestellar cores masses or core mass function (CMF) in L1688. The resulting CMF (blue histogram in Fig. 4) is very similar in shape to the initial mass function (IMF), thus confirming the role of prestellar cores as precursors of protostars (cf. Motte et al.1998). The star-formation efficiency in individual cores, $\epsilon_{\text {core }}$, is estimated to be $0.5 \pm 0.1$ in this region, based on the location of the peak of the prestellar CMF (Fig. 4) compared to that of the IMF. 


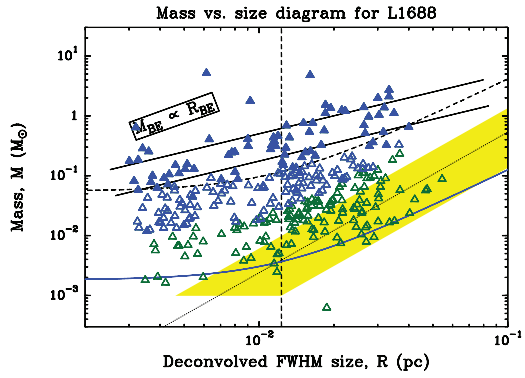

Figure 1. Mass vs. Size diagram for the starless dense cores found with Herschel in L1688. Robust prestellar cores are shown by filled blue triangles and candidate prestellar cores by open blue triangles. Very low-mass unbound starless cores (open green triangles) are detected but may be transient.

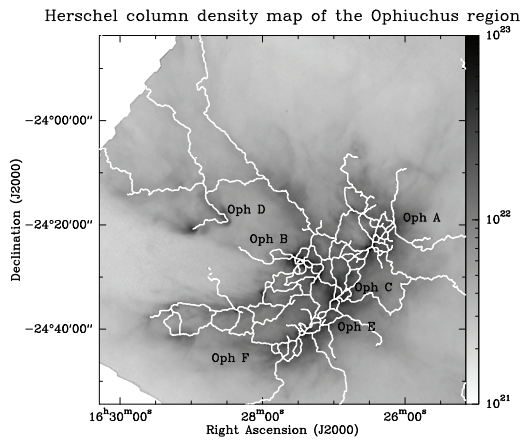

Figure 3. Herschel column density map of L1688 (Ladjelate et al.in prep). The white lines trace the filaments detected with DisPerse (Sousbie 2011). Most prestellar cores are formed along these lines, in the densest filaments.

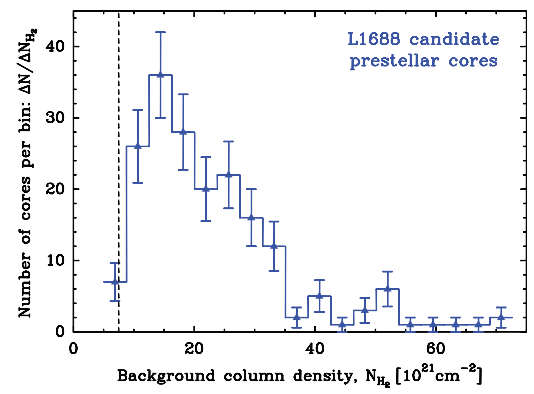

Figure 2. Number of prestellar cores per background column density bin. Most prestellar cores are formed above $\mathrm{A}_{V}=8$. This is similar to the core formation threshold found in the Aquila cloud (Könyves et al.2015).

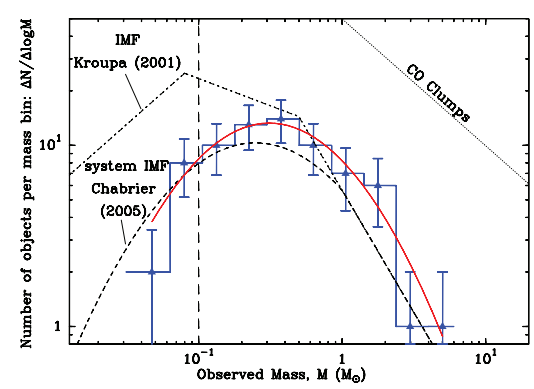

Figure 4. Prestellar core mass function (CMF) in L1688 (in red). The peak of the prestellar CMF appears to be close to $0.3 \mathrm{M}_{\odot}$ in L1688. Simulations suggest that the actual peak is at $\sim 0.5 \mathrm{M}_{\odot}$ because the derived masses are underestimated by $\sim 50 \%$ on average (cf. Appendix B of Könyves et al.2015).

\section{References}

André, P., Di Francesco, J., Ward-Thompson, D., et al. 2014, Protostars and Planets VI, 27 Könyves, V., André, P., Men'shchikov, A., et al. 2015, A\&GA, arXiv:1507.05926

Men'shchikov, A., André, P., Didelon, P., et al. 2012, A\&AA, 542, A81

Men'shchikov, A. 2013, A\&\&A, 560, A63

Montmerle, T., Koch-Miramond, L., Falgarone, E., \& Grindlay, J. E. 1983, ApJ, 269, 182

Motte, F., André, P., \& Neri, R. 1998, A\& A, 336, 150

Sousbie, T. 2011, MNRAS, 414, 350

Wilking, B. A., Lada, C. J., \& Young, E. T. 1989, ApJ, 340, 823 